\title{
A SURVEY STUDY OF FRACTIONAL ORDER CONTROL TECHNIQUES
}

\author{
Dr. Layla H.Abood \\ Department of Control and Systems Engineering \\ University of Technology \\ Baghdad, IRAQ
}

\begin{abstract}
- nowadays, there is an interest for using fractional calculus in many applications and different researches are presenting in many fields. Control engineering problems are also solved using fractional calculus because it analyzes real system dynamics accurately. In this paper, a review study that present different researches using factional order controllers(FOC). The fractional order PID controller are most widely used in the application of control systems due to its simple structure and easy implementation, then the researcher use different techniques mixed with fractional calculus like fractional order neural network, fractional order fuzzy logic controller, fractional order sliding mode controller and finally fractional order backstepping controller, the recently advances is demonstrated in this review study.
\end{abstract}

Keywords - fractional calculus, fractional order PID controller, fractional order neural network, fractional order fuzzy system, fractional order sliding mode controller, fractional order backstepping controller.

\section{INTRODUCTION}

Fractional calculus is the generalized form for a classical integer-order form which have includes two operators the integral and the derivative operator in fractional orders, factional calculus has acquired comprehensive attention in recent years in many fields like engineering, physics, and chemistry systems due to the best and accurate system dynamic descriptions [1].The fractional calculus is the improvement of the derivative and integral fractional (noninteger) order, in fractional calculus the most important operator is ${ }_{\mathrm{a}} D_{\mathrm{t}}{ }^{\mathrm{C}}$ the variable a and $\mathrm{t}$ are the lower and upper values for the fractional operation and $\alpha$ represents the differintegral order, then the differential operator will be as shown below:

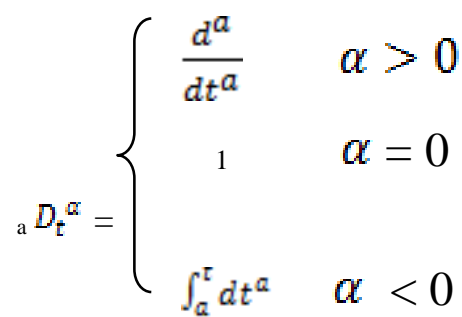

The fractional differintegral have three essential definitions: Caputo formula, Riemann Liouville (RL), and Grünwald Letnikov (GL)[2]. Fractional calculus becomes in the list of the recent trends in control systems that used in modeling description and the control design besides its ability to improve closed-loop systems performance and robustness [3]. Different types of fractional order controllers are adopted to control many systems; some fractional order controllers with a review of related researches are listed here. In this paper, fractional calculus is considered as a useful method used in these different fractional controllers.

\section{FRACTIONAL ORDER PID CONTROLLER}

In 1994 Podlubny [4] presents the most common FOC, the fractional order $\mathrm{PID}$ or $\mathrm{PI}_{\mu} \mathrm{D}_{\lambda}$ where $\mu$ and $\lambda$ represents the order of the integrator and a differentiator. Various researches adopt this type of FOC, in [5] an optimal FOPID is used for controlling the speed of brushless DC motor, desired values and efficient response are obtained due to the use of FOPID with the Bat algorithm for find the controller optimal values, the controller controlled the system with no load, 50\% load and $100 \%$ load which gives efficient performance and best controllability based on system characteristics like settling time, rise time, peak time and finally the error value at steady state for different conditions.

In [6] author use FOPID controller for controlling AVR system then compare its response with classical PID controller, the simulation results reflects the superiority of FOPID controller, in [7] author a study uses implementable FO PID controllers for controlling a system with of first order time delay system, an algebraic equations is used based on two fitness functions ISE and ISTE, values of fitness 


\section{International Journal of Engineering Applied Sciences and Technology, 2021 \\ Vol. 6, Issue 2, ISSN No. 2455-2143, Pages 1-4 \\ Published Online June 2021 in IJEAST (http://www.ijeast.com)}

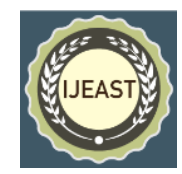

functions are minimized when compared with classical PID controllers.

In [8] author adopts three controllers a classical PID controller tuned using Ziegler-Nichols, classical PID controller tuned using ant colony optimization method and finally FOPID controller tuned using ant colony optimization for controlling the speed of a DC motor, the simulation results show the efficient behavior of FOPID in minimizing the error value between actual and desired response at steady state with minimum overshoot and settling time, in [9] a FOPID controller is used for controlling a model of third-order (a permanent magnet synchronous motor), parameters of the controller are obtained analytically that achieves an efficient performance when compared with optimal FOPID and Bode shaping FOPID controllers.

\section{FRACTIONAL ORDER NEURAL NETWORK CONTROLLER}

The fractional calculus has been used in different application because it gives an efficient instrument for the characterization of memory and inborn properties of different fields and processes. Due to these properties, fractional calculus was adopted in the field of neural networks. The fractional-order differentiation supplies neurons with a computational potency that reflects a good information processing, and the fractance in fractional calculus will used instead of the common capacitance used in the continuous time neural network, this will generate the fractional-order neural network [10].

Different studies were utilized this type of fractional order controller, in[11] the author adopts FOPID controller with auto tuning neuron for controlling pulp consistency control system and the neural network used is the Radial basis Function (RBF) type then a comparison with traditional PID and self tuning PID (PIDNN) is done to show the effeteness of FOPIDNN, the simulation results reflects the superiority of FOPINN in drive the system to efficient adaptability and make it more robust. In [12] a back- propagation neural network is proposed with a structure of four input layers and five hidden layers and three output layers then a sigmoid activation function is used, a self tuning algorithm is applied to calculate the gains of fractional order PI controller, the flexibility and ability to find parameters based on self tuning method introduce an efficient response if compared with normal type of FOPI controller.

In [13] a robust structure of FONN is utilized by combining $\mathrm{H}-\infty$ with controller since it is affected by time delay, then a novel control technique is utilized by use a memristor then Mittag-Leffler method is used for test stability that applied to different numerical relations, in [14] a FOPID controller is tuning based on using five back propagation neural networks and Kalman filter algorithm also used as an emulator for identification the behavior of the plant, two types of plants are used one has fast dynamics and other with time delayed system then the behavior for both systems used showed the accurate and robust response with satisfied results.

In [15] the design of a back propagation neural network fractional controller is designed based on Hausdorff derivative and integral, which is introduced to PID controller and, the tuning process for all controller parameters and order is done using Borges derivative which enhance the optimization process and fastly obtained the suitable values for reaching to desired response and finally a comparison with PIDNN that show the improvement appear on the response speed with accurate and stable behavior.

\section{FRACTIONAL ORDER BACKSTEPPING CONTROLLER}

Fractional calculus techniques are characterized as a powerful tools for achieve robust performance in control system. Hybridization between Fractional order calculus and backstepping controller enhance and improve the system response and some of the recent papers will demonstrated, in [16] author propose an adaptive Fractional Order Backstepping Controller (FOABC) for controlling DOF robotic manipulator and the stability is tested based on fractional Lyapunov method, a set of various experiment is applied on system to see controller ability in achieve the tracking performance based on different desired input also an external disturbance is applied, the controller led the system to a satisfied response in tracking issue and in facing disturbances issue.

In [17] an adaptive controller is proposed for controlling ferroresonance system based on FOABC, FO Lyapunov stability method is used and the update and virtual control laws is test at each stage to achieve an enhanced behavior in controlling the ferroresonance system with effective desired response. $\operatorname{In}[18]$ a novel combination between backstepping controller and $\mathrm{n}$ fractional order chaotic system, the system is tested with new lemma and Lyapunov method in systematic manner, simulation and graphical results showed that this new scheme with its easy implementation are able to achieve good synchronization for the FO chaotic plants.

\section{FRACTIONAL ORDER FUZZY LOGIC CONTROLLER}

Fractional order calculus can be mixed with classical Fuzzy controller by make the error and its fractional derivative is the input for the controller and its output integrated with the fractional order. Different studies apply this controller, in [19] FO fuzzy PD + I structure is proposed to control a nonlinear robotic manipulator and the tuning method used for obtain the controller gains is genetic algorithm numerical results show the superiority of FOFPD + I type as compared with FDP+I 


\section{International Journal of Engineering Applied Sciences and Technology, 2021 \\ Vol. 6, Issue 2, ISSN No. 2455-2143, Pages 1-4 \\ Published Online June 2021 in IJEAST (http://www.ijeast.com)}

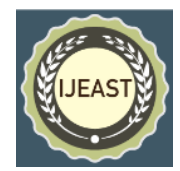

and classical PID controllers also different robustness analysis are applied to the system like model uncertainty, reject disturbance and changing the reference trajectory, the controller faced all these matters and drive the system to achieve robust and efficient response when compared with different controllers.

In [20] a FFOPID scheme is proposed to control the pneumatic pressure system by use the error and its derivative as inputs and then find the gain of the PID controller and these gains will update the parameters of FOPID controller. A comparison between classical PID and FOPID and FFOPID is done which reflects robustness and adaptability of FFOPID controller, in [21] a hybrid structure is present consists of $\left(\mathrm{FI}+\mathrm{FI}^{\alpha}+\mathrm{FD}^{\beta}\right)$ for controlling the nonlinear power system with the benefits of using a hybrid optimization method mixing virus colony search (VCS) and artificial bee colony (ABC), numerical analysis showed that the suggested controller make the system response stable and robust with lower fall time and frequency oscillations when compared with classical PID and FOPID controllers. In [22] a FF Differential Equation (FFDE) is used with corona virus infection system by using fuzzy Laplace transform and Adomian decomposition technique, then compare the results obtained from suggested controller and normal results, as shown from results it gives excellent result in finding the problem dynamics even it suffer from uncertainty.

\section{FRACTIONAL ORDER SLIDING MODE CONTROLLER}

Fractional Order Sliding Mode Controller (FOSMC) is the generalized form of classical Sliding Mode Controller (SMC) which is combined with fractional calculus to generate FOSMC and this is done when state variables. Is appeared as FO derivative and/or integral form in the sliding surface, different structure is proposed in the recent studies will demonstrate, in [23] author suggests a FOSMC combined with Linear Quadratic Regulator (LQR) which is adopted to stabilized the nonlinear system which the robustness is achieved by FOSMC, the results obtained showed efficient and robust behavior of the system despite of uncertainty issue.

In [24] a fractional order backstepping controller is combined with SMC is proposed to regulate blood glucose for diabetic patients by minimizing the needed time for glucose to reach to its desired value, simulation results showed that the robustness and convergence of system state variable is achieved due to the use of SMC and BS controller, in [25] the FOSMC is proposed based on the use of time variant sliding surface and Lyapunov method is used to test the controller stability, simulation are done for two model robot manipulator and mass-spring-damper with external disturbances and system uncertainties than the suggested controller is compared with classical SMC, an improvement is appeared clearly in the fast settling time and reduced the error to obtain stable and robust response.
In [26] a FOSMC is proposed with spherical robot with input saturation and a filter is adopted to achieve good control response to defeat the input saturation. The stability is tested based on Lyapunov method and then it is compared with the traditional SMC, the adjustment time become shorter and no overshoot appeared in its response.

\section{CONCLUSION}

From the papers demonstrated in this study it can be seen that FOPID controller is more robust than classical PID controller this is due to the two added fractional parameter which help in reaching to desired value faster than the classical PID, the use of self-tuning method in neural fractional order PID give robust to the system in facing model uncertainties and different disturbances. In using FOSMC the chattering problem will reduced and the Lyapunov stability method is applied in the two FOSMC \& SMC types. In using FOABC will ensure the system convergence and achieve robust behavior in facing disturbances and model uncertainties.

\section{REFERENCE}

[1] El El-Khazali, Reyad. (2013). Fractional-order PI $\lambda \mathrm{D} \mu$ controller design." Computers \& Mathematics with Applications vol.66, no.5, pp.639-646.

[2] Bhookya J., Jatoth R.K . (2019). Optimal FOPID/PID controller parameters tuning for the AVR system based on sine-cosine-algorithm. Evolutionary Intelligence vol.12, no.4, pp. 725-733.

[3] Folea S., Muresan C. I. , Keyser, R.D. \& Ionescu, C. M. (2015). Theoretical analysis and experimental validation of a simplified fractional order controller for a magnetic levitation system". IEEE transactions on control systems technology, 24(2), pp.756-763.

[4] Podlubny, I., (1994). Fractional-Order Systems and Fractional-Order Controllers . Institute of Experimental Physics, Slovak Academic. UEF-03-94.

[5] Vanchinathan, K., \& Valluvan, K. R. (2017). A metaheuristic optimization approach for tuning of fractional-order PID controller for speed control of sensorless BLDC motor. Journal of circuits, systems and computers, http://dx.doi.org/10.1142/S0218126618501232.

[6] Roy, S., Chakraborty, S., \& Mondal, U. (2018). Fractional-order controller for automatic voltage regulator. In IEEE Applied Signal Processing Conference (ASPCON) pp. 4-8.

[7] Ashjaee, M. \& Tavazoei, M. S. (2019). Tuning the Implementable Structures of Fractional-Order PID Controllers for Control of FOPDT Processes. Scientia Iranica., http://dx.doi.org/10.24200/sci.2019.51703.2321. 


\section{International Journal of Engineering Applied Sciences and Technology, 2021 \\ Vol. 6, Issue 2, ISSN No. 2455-2143, Pages 1-4 \\ Published Online June 2021 in IJEAST (http://www.ijeast.com)}

[8] Yadav, J., Kumar, P., Gupta, N., \& Kaur, M. (2020). Speed Control of DC Motor using FOPID Controller based on Ant Colony Optimization. Artificial \& Computational Intelligence, Vol.1,no.3.

[9] Zheng, W., Luo, Y., Chen, Y.Q., \& Wang, X. (2021). A Simplified Fractional Order PID Controller's Optimal Tuning: A Case Study on a PMSM Speed Servo. Entropy, https://doi.org/10.3390/e23020130.

[10] Gu, Y., Yu, Y., \& Wang, H. (2017). Synchronizationbased parameter estimation of fractional-order neural networks. Physica A: Statistical Mechanics and its Applications.

http://dx.doi.org/10.1016/j.physa.2017.04.124.

[11] Jaun S.W. \& Wei T. (2016). A neural network fractional order PID controller for FOLPD process. In IEEE 35th Chinese Control Conference (CCC) pp. 10459-10463.

[12] LI W. (2008) .Design and Implement of Neural Network Based Fractional-Order Controller. Intelligence and Automation. Springer, Berlin, pp. 471-479.

[13] Huang, H., Yuan, X., \& Mo, L. (2019). Memristor-based $\mathrm{H} \infty$ Synchronization Control for Fractional-order Neural Networks with Time-delays. In International Conference on Modeling, Simulation, Optimization and Numerical Techniques (SMONT 2019), pp. 184-188.

[14] Moghadam M. P., Saryazdi, M. G., Loghman, E., Kamali, A., \& Bakhtiari-Nejad, F. B. (2020). Development of neural fractional order PID controller with emulator. ISA transactions, https://doi.org/10.1016/j.isatra.2020.06.014.

[15] Gao Z. (2021). A Tuning Method via Borges Derivative of a Neural Network-Based Discrete-Time FractionalOrder PID Controller with Hausdorff Difference and Hausdorff Sum. Fractal and Fractional , https://doi.org/10.3390/fractalfract5010023.

[16] Nikdel, N., Badamchizadeh, M., Azimirad, V., \& Nazari, M. A. (2016). "Fractional-order adaptive backstepping control of robotic manipulators in the presence of model uncertainties and external disturbances". IEEE Transactions on Industrial Electronics, https://doi.org/10.1109/TIE.2016.2577624.

[17] Wang, Y., Liu, L., Liu, C., Zhu, Z., \& Sun, Z. (2018). "Fractional-order adaptive backstepping control of a noncommensurate fractional-order ferroresonance system. Mathematical Problems in Engineering, https://doi.org/10.1155/2018/8091757.

[18] Yadav, V. K., \& Das, S. (2019)." Combination synchronization of fractional order n-chaotic systems using active backstepping design". Nonlinear Engineering, vol.8, no.1, pp597-608.

[19] Chhabra, H., Mohan, V., Rani, A., \& Singh, V. (2019). Robust nonlinear fractional order fuzzy PD plus fuzzy I controller applied to robotic manipulator. .Neural
Computing and https://doi.org/10.1007/s00521-019-04074-3.

[20] Al-Dhaifallah, M., Kanagaraj, N., \& Nisar, K. S. (2018). Fuzzy fractional-order PID controller for fractional model of pneumatic pressure system. Mathematical Problems in Engineering, https://doi.org/10.1155/2018/5478781.

[21] Eslami, M., Shayesteh, M. R., Pourahmadi, M., \& Ayatollahitafti, V. (2019). Optimal design of fractional order fuzzy PID controller using an intelligent hybrid algorithm for nonlinear power system . Journal of Intelligent \& Fuzzy Systems, https://doi.org/ 10.3233/JIFS-182918.

[22] Ahmad, S., Ullah, A., Shah, K., Salahshour, S., Ahmadian, A., \& Ciano, T. (2020). Fuzzy fractional-order model of the novel corona virus. Advances in difference equations, https://doi.org/ 10.1186/s13662-020-02934-0.

[23] Zhang, D., Cao, L., \& Tang, S. (2017). Fractional-order sliding mode control for a class of uncertain nonlinear systems based on LQR. International Journal of Advanced Robotic Systems, https://doi.org/10.1177/1729881417694290.

[24] Vakili, S., \& ToosianShandiz, H. (2019)." Fractional Order Glucose Insulin System Using Fractional BackStepping Sliding Mode Control." International Journal of Nonlinear Analysis and Applications, https://dx.doi.org/10.22075/IJNAA.2019.4067.

[25] Eray O., Tokat, S. (2020). The design of a fractional-order sliding mode controller with a time-varying sliding surface. Transactions of the Institute of Measurement and Control, https://doi.org/10.1177/0142331220944626.

[26] Zhou T., Xu Y. \&Wu. B. (2020). Smooth Fractional Order Sliding Mode Controller for Spherical Robots with Input Saturation. Applied Sciences, http://dx.doi.org/10.3390/app10062117. 